\title{
Semi-Supervised Rough Fuzzy Clustering for Brain MRI Segmentation
}

\author{
Nur Alom Talukdar ${ }^{1}$, Anindya Halder ${ }^{2}$ \\ Department of Computer Applications, School Of Technology, North-Eastern Hill University ${ }^{1}$ \\ Email: nuralom1988@gmail.com ${ }^{1}$, anindya.halder@gmail.com ${ }^{2}$ (corresponding author)
}

\begin{abstract}
In modern medical diagnosis, automated system plays a major role for proper investigation of diseases and giving accurate therapy. Mostly segmentation is the first step in almost all automated diagnosing systems where magnetic resonance imaging (MRI) is concerned. The use of brain MRI is in its pick in the recent time for diagnosing brain related disease. In this article, a new semi-supervised rough fuzzy C-means (SSRFCM) clustering algorithm is proposed by using a small amount of labeled pixels in the clustering process for the accurate segmentation of brain MRI which is a judicious amalgamation of the concept of fuzzy sets and rough sets. Where, fuzzy set and the rough set concept in the process of clustering manage the uncertainty, indiscernibility, vagueness and overlappingness of different brain regions normally present in brain MRI. On the other hand, constraints in the form of labeled pixels are used here to initialize and guide the clustering process to achieve accurate segmentation of brain regions. Experiments are carried out on varieties of benchmark real life as well as synthetic brain MRI data sets. Performance of the proposed SSRFCM algorithm is tested with widely used other counterparts clustering based algorithms. Experimental results of the proposed method are evaluated using overall segmentation accuracy, jaccard, and dice coefficient. Segmentation results show that the proposed method outperformed the counterpart algorithms in terms of segmentation accuracy. Paired $t$-test confirms the statistical significance of the improved segmentation accuracy in support of the proposed method compared to other clustering based methods with a convincingly small amount of supervision.
\end{abstract}

Index Terms- Brain MRI; Segmentation; Clustering; Rough Set; Fuzzy Set; Semi-Supervised.

\section{INRODUCTION}

In recent time, brain tissue segmentation from brain magnetic resonance imaging (MRI) draw the attention of the researchers in modern medical sciences and in modern medical diagnosing due to its uses in different real world problems such as identification of tumor, edema, or any new abnormalities related to brain [12]. MRI can produce multimodal images of different regions of brain with a range of contrasts based on the different MR modalities such as, T1, T2, and proton density because of which researchers choose the MRI over other tools such as X-ray, CT scan for brain segmentation [1], [3-4]. Brain segmentation can be defined as the process of grouping a brain MRI into separate parts according to different purposes and objectives based on some internal characteristics of brain tissues [3].

Quality and accurate segmentation play a vital role to the performance of automated systems [5-8]. In machine learning, brain MRI segmentation can be taken as a clustering problem where similar regions of the MRI are partitioned into one group and dissimilar regions are partitioned into different group (cluster) [6]. As the structure of human brain and its tissue boundaries are vague, uncertain, indiscernible and overlapping in nature, so to deal with these fuzziness concepts is borrowed in to the clustering process which forms the concept of membership of a pixel (data point) to a given cluster known as fuzzy clustering $[6,8]$. A variety of fuzzy clustering method as well as non-fuzzy clustering methods have been applied for the segmentation of brain MRI such as thresholding based methods [9-10], region-growing based [11], edge detection based method [12,13], fuzzy clustering/machine-learning/pattern recognition based methods [14-19] etc. Good reviews of brain MRI segmentation methods can be referred in [20-22]. Amongst all fuzzy clustering techniques, semisupervised fuzzy clustering generally performs better $[23,24]$, given that extra added information like pair wise constraint (must-link and cannot-link constraints between points in a dataset) or class labels or a predefined membership values are supplied in the clustering process [24].

In this article, for brain MRI segmentation the second technique i.e., providing some class labels (i.e., some labeled information) is used to formulate the problem by means of semi-supervised clustering with the goal to enhance the segmentation performance in terms of accuracy than the existing techniques.

The rest of the article is organized as follows. In Section 2 background of the fuzzy sets and rough sets are briefly discussed. The proposed technique SSRFCM is discussed in Section 3. The datasets, compared methods and cluster validity measures are 


\section{Available online at www.ijrat.org}

discussed under experimental setup in Section 4. In Section 5 result analysis and discussions are summarized. Finally, Section 6 highlights the conclusion and future direction of works.

\section{BACKGROUD OF THE RELATED STUDIES}

The proposed method is based on judicious amalgamation of rough sets and fuzzy sets, therefore, in this section a brief overview of the fuzzy sets and rough sets have been described.

\subsection{Fuzzy sets}

Let, universe $U$ is a finite and nonempty set. A fuzzy set [25] $F$ of $U$ is a mapping from $U$ into the unit interval $[0,1] ; \mu_{F}: U \rightarrow[0,1]$ where, for each $x \in U$, we call $\mu_{F}(x)$ the membership degree of $x$ in $F$. Then, a fuzzy set can be considered as a mathematical representation of a vague concept described linguistically. The support of fuzzy set $F$ is the crisp set that contains all the elements of $U$ that have a nonzero membership value in $F$. A function mapping all the elements in a crisp set into real numbers in $[0$, 1] is called a membership function [25]. Greater value of the membership function signifies the higher degree of the membership. It means how closely an element is similar to an ideal element. Membership functions can represent the uncertainty using some particular functions. These functions convert the linguistic variables into numerical calculations by setting some parameters [25]. The fuzzy decisions can be made.

\subsection{Rough sets}

Let, $\mathrm{Z}=(U, R)$ is an approximation space, where $U$ represents a finite set (the universe) and $R$ represents an equivalence relation on the universe $U$. For a given subset of attributes, the set of objects may be divided in equivalence classes. For each subset $X \subseteq U$, it may define a lower approximation $(\underline{B} X)$ and an upper approximation $(\bar{B} X)$ as follows.

$$
\begin{gathered}
\underline{B} X=\bigcup_{X_{i} \in X} X_{i} \\
\bar{B} X=\bigcup_{X_{i} \cap X \neq \emptyset} X_{i}
\end{gathered}
$$

$\underline{B} X$ is defined as the union of all equivalence classes which are fully included in $\mathrm{X} . \bar{B} X$ is defined as the union of all equivalence classes which have a nonempty intersection with that of $X$. A rough set proposed by Pawlak [26,27] is a formal approximation of the crisp set in terms of the lower and upper approximation.

\section{SEMI-SUPERVISED ROUGH FUZZY C- MEANS (SSRFCM) CLUSTERING}

The main aim of the proposed method is to segment brain MRI more accurately. Usually, the boundaries of different brain regions are vague, uncertain, and indiscernible and overlapping in nature. To deal with these characteristics the proposed method is introduced with the judicious incorporation of the concept of fuzzy set and rough set in semi-supervised mode with a little amount of supervision in the form of labeled data in the clustering process. In Fuzzy C-Means (FCM) $[28,29]$ clustering it works by optimizing the objective function which can be defined as:

$$
J_{F C M}=\sum_{i=1}^{C} \sum_{k=1}^{N} \mu_{i k}^{m}\left(\overrightarrow{x_{k}}-\overrightarrow{v_{l}}\right)^{2} ;
$$

The updations of memberships $\left(\mu_{\mathrm{ik}}\right)$ and the cluster centers $\left(\overrightarrow{v_{l}}\right)$, at each iteration are done respectively as follows:

$$
\begin{aligned}
\mu_{i k} & =\frac{\left(\left(\overrightarrow{\boldsymbol{x}_{\boldsymbol{k}}}-\overrightarrow{\boldsymbol{v}_{\boldsymbol{\imath}}}\right)^{2}\right)^{\frac{1}{1-m}}}{\sum_{i=1}^{C}\left(\left(\overrightarrow{\boldsymbol{x}_{\boldsymbol{k}}}-\overrightarrow{\boldsymbol{v}_{\boldsymbol{\imath}}}\right)^{2}\right)^{\frac{1}{1-m}}} ; \\
\overrightarrow{v_{\boldsymbol{l}}} & =\frac{\sum_{k=1}^{N} \mu_{i k}^{m} \overrightarrow{\boldsymbol{x}_{\boldsymbol{k}}}}{\sum_{k=1}^{N} \mu_{i k}^{m}} ;
\end{aligned}
$$

Where, $m$ represents the fuzziness index, $C$ is the number of clusters and $N$ is the number of pixels (data patterns).

After the integration of the concept of rough set into FCM, fuzzy clusters are treated as rough clusters and can be defined with the concept of lower approximation $\left(\underline{B} X_{i}\right)$ and upper approximation $\left(\bar{B} X_{i}\right)$ and the assignment of a pixel in $\underline{B} X_{i}$ and $\bar{B} X_{i}$ using the fuzzy membership is done as, (1) A pixel $\vec{x}_{k}$ lies in at most one $\underline{B} X_{i}$ of a (generic rough) cluster. (2) If a pixel $\vec{x}_{k} \in \underline{B} X_{i}$ of a rough cluster $X_{i}$, then it must also belongs to the upper approximation i.e., $\vec{x}_{k} \in \bar{B} X_{i}$ of the same cluster. (3) If a pixel $\vec{x}_{k}$ is not a part of $\underline{B} X_{i}$ of any rough cluster then it belongs to $\bar{B} X_{i}$ of multiple rough clusters. Therefore, the amalgamation of rough set (in FCM) requires additional change in the cluster centre computation (i.e., in Eq. 3) which can be defined as in Eq. 4. 


\section{Available online at www.ijrat.org}

Where, $w_{\text {low }}$ and $w_{u p}$ are control parameters to assign

pattern). $\mu_{i k}^{u}$, is the membership of unlabeled pattern (pixels) with respect to cluster center $\vec{v}_{i}$ and unlabeled

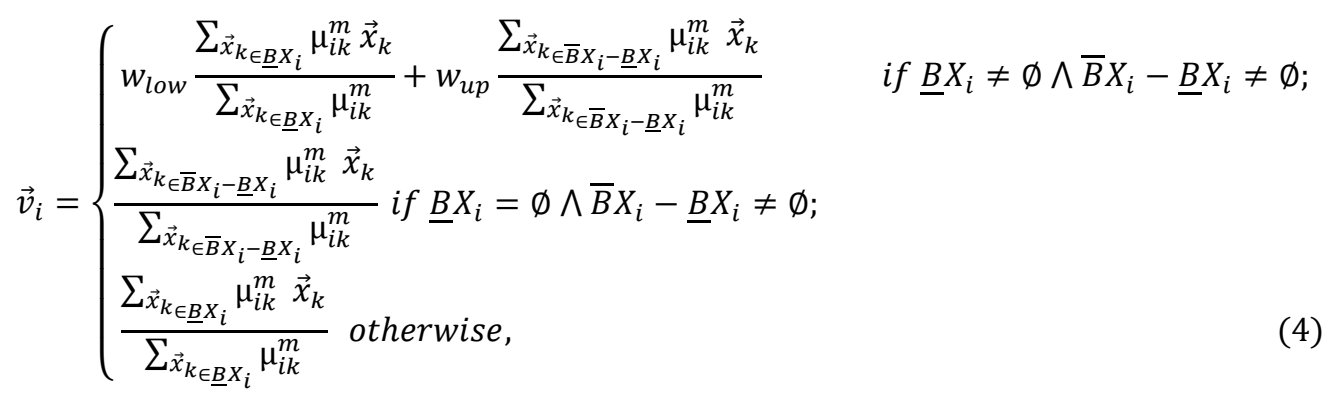

weights for pixels (points) belonging to $\bar{B} X_{i}$ and $\underline{B} X_{i}$ value. Usually $w_{\text {low }} \in[0.5,1]$ has higher weight than $w_{u p}$, and $w_{\text {low }}+w_{\text {up }}=1$.

Now, in the clustering process some label pixels are supplied to enhance the performance of the clustering process which turns the clustering process into a semisupervised method. It's worth noting that in the traditional clustering process the initial clusters are chosen randomly and if the initial cluster centers are not chosen accurately then it may lead to an inaccurate result which lowers down the performance of the clustering process. To overcome the problem of choosing initial cluster center small number of label pixels are supplied to guide the clustering process for choosing better initial cluster centers. Where the initial cluster centers will be choosing from the mean of those labeled pixels instead of choosing randomly which in turn enhance the performance of the clustering process significantly.

Let, a data set $X$ can be describe as $X^{1} \cup X^{u}$. Where, $X^{u}$ contain the unlabeled data patterns (pixels) and $X^{l}$ contain the labeled data patterns (pixels). where, $X^{u} \subseteq$ $\mathrm{X}, X^{l} \subseteq \mathrm{X}$ and $\vec{x}_{k}{ }^{u} \in X^{u}, \vec{x}_{k}^{l} \in X^{l}$.

After the addition of some labeled pixels in the clustering process it needs some modification in the cluster center, $\left(\vec{v}_{i}\right)$ updation function and can be defined as in Eq. 5.

Here, $\mu_{i k}^{l}$, is the membership of the $k^{\text {th }}$ labeled pattern (pixels) is always assumed to be 1 (one) for belonging the cluster $i$ (from prior label information about the pattern $\vec{x}_{k}$. Since in the proposed method constraint policy is applied therefore, throughout the clustering process the membership value for the labeled pattern (pixels) remain unchanged.

\section{EXPERIMENTAL SET UP}

\subsection{Brain MRI datasets used}

The proposed method SSRFCM is simulated with a variety of benchmark synthetic as well as real life brain MRI datasets. Synthetic datasets considered from BrainWeb (BW) [30] brain MRI database (freely available online at: http://brainweb.bic.mni.mcgill.ca/brainweb) and the real life datasets considered from widely used IBSR [31] brain MRI database (freely available online at: https://www.nitrc.org/projects/ibsr). Considering the importance of the various region of brain for the present study the number of regions (cluster) is considered to be three (3) viz., White Matter (WM), Gray Matter (GM), and Cerebrospinal Fluid (CSF). Size of the real life IBSR dataset is $181 \times 256$ whereas; the size of the synthetic BarinWeb dataset is $217 \times 18$. Simulations are carried out in Windows environment in Intel core i5 processor having 4GB of RAM in Matlab R2014b.

\subsection{Methods compared for the study}

Performance of the proposed method SSRFCM is compared with two commonly used popular partitive clustering based segmentation algorithms viz., (i) Fuzzy C-means (FCM) [28, 29], and (ii) Rough fuzzy

$$
\text { if } \underline{B} X_{i} \neq \emptyset \wedge \bar{B} X_{i}-\underline{B} X_{i} \neq \emptyset \text {; }
$$

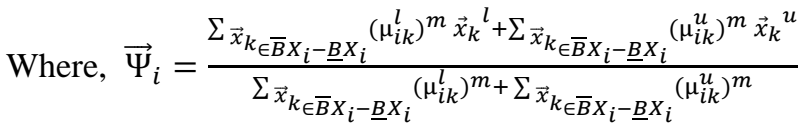

$$
\begin{aligned}
& \vec{X}_{i}=\frac{\sum_{\vec{x}_{k_{\in} X_{i}}}\left(\mu_{i k}^{l}\right)^{m} \vec{x}_{k}^{l}+\sum_{\vec{x}_{k_{\in} \underline{B} X_{i}}}\left(\mu_{i k}^{u}\right)^{m} \vec{x}_{k}^{u}}{\sum_{\vec{x}_{k_{\in} \underline{B} X_{i}}}\left(\mu_{i k}^{l}\right)^{m}+\sum_{\vec{x}_{k_{\in B} X_{i}}}\left(\mu_{i k}^{u}\right)^{m}}
\end{aligned}
$$


International Journal of Research in Advent Technology, Vol.7, No.3, March 2019 E-ISSN: 2321-9637

\section{Available online at $w w w . i j r a t . o r g$}

C-means (RFCM) [4, 32, 33] as the proposed method is a partitive clustering technique

\subsection{Clustering validity measures used}

To evaluate the performance of the proposed SSRFCM method a variety of segmentation validity indices [34] viz., (i) overall segmentation accuracy (OSA) [17], (ii) Jaccard coefficient [17], and (iii) Dice coefficient [17] are used, which are discussed below:

\subsubsection{Overall segmentation accuracy (OSA:}

It is the ratio of correctly identified pixels to the total pixels present in the brain MRI can be defined as,

$$
O S A=\frac{A}{B}
$$

Where, $A$ represents the correctly identified pixels, and $B$ represents the total pixels present in the brain MRI.

\subsubsection{Jaccard coefficient:}

It is the ratio of intersection to the union of the original MRI and Segmented image can be defined as,

$$
J=\frac{S(A \cap B)}{S(A \cup B)}
$$

Where, A represents the segmented output, B represents the original brain MRI and $S$ represents the summation function. The value of $J$ lies between 0 and 1. Higher the value of $J$ better is the segmentation.

\subsubsection{Dice coefficient:}

Dice coefficient can be derived from jaccard coefficient, which defines a similarity function of the original MRI and the segmented output and defined as:

$$
D=2 \times \frac{J}{1+J}
$$

\section{RESULTS ANALYSIS AND DISCUSSION}

Experiments have been performed on a number of variety benchmark brain MRI from BrainWeb and IBSR dataset. For the purpose of reporting only few representative experimental results in terms of overall segmentation accuracy, jaccard and dice validity measures along with the corresponding segmented results (brain MRI outputs) obtained by the proposed method and the counterpart methods are shown for the BrainWeb MRI brain data sets (Fig. 1) as well as for IBSR brain MRI data sets (Fig. 2) in Table 1. The ground truth images for both the BrainWeb dataset as well as IBSR dataset are shown in the second column of Fig. 1 for brain MRI Z93 and and Z100 and in Fig. 2 for brain MRI IBSR 155 and IBSR 167.

The best results in terms of validation measures (overall segmentation accuracy (OSA), jaccard, and Dice) for each data are shown bold for the subsequent method in Table 1.
In Fig. 3 the segmented results are summarized for brain MRI datasets considered from BrainWeb, where the first column represents the segmented images of BW Z93 and second column represents BW Z100 by the proposed method along with the counterpart methods.

Similarly, in Fig. 4 the segmented results are summarized for brain MRI datasets considered from IBSR, where the first column represents the segmented images of IBSR 155 and the second column represents IBSR 167 by the proposed method along with the counterpart methods.

Similar results are also found for other datasets. In summary the proposed semi-supervised method with little supervision outperformed the counterpart algorithm in terms of segmentation quality as well as from the validity indices.

The Box-and-Whisker plots [35] (also known as Boxplot) of overall percentage accuracy found by the proposed method as well as the counterpart methods are shown in Fig. 5. Where, (a) is the Box-plot obtained by the methods on brain MRI data set IBSR 155, (b) IBSR 167, (c) BrainWeb Z93, and (d) BrainWeb Z100. From the Box-plots it can be observed that the Box-plot obtain by the proposed method is relatively dense compare with the counterpart methods and the standard deviation is less as well as the mean of the segmentation accuracy is high. This shows that segmentation accuracy of the proposed method outperforms the counterpart methods.

Experimental results are statistically validated using paired $t$-test [36] 5\% level of signicance. The null hypothesis of the analysis believes that there is no major dissimilarity among the results obtained by the proposed method and the counterpart methods. Paired t-test results found by the proposed method versus other compared methods in terms of $p$-score are reported in Table 2 . Results of $t$-test are statistically important (at 5\% level of significance) if the subsequent $p$ value is equal or less than 0.05 , signifying that the null hypothesis is rejected. From the results observed in Table 2, it can be concluded that out of the total test performed (except one case) the proposed method dominates the counterpart methods statistically. This proofs the superiority of the proposed method. 
International Journal of Research in Advent Technology, Vol.7, No.3, March 2019 E-ISSN: 2321-9637

Available online at www.ijrat.org

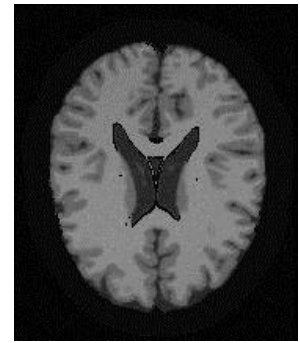

(a)

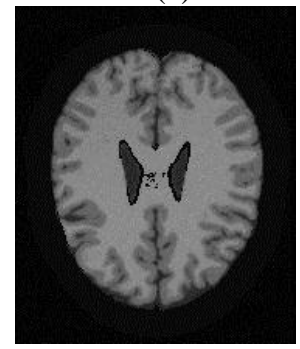

(c)

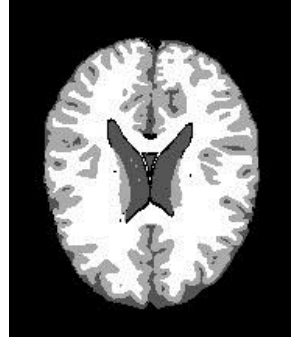

(b)

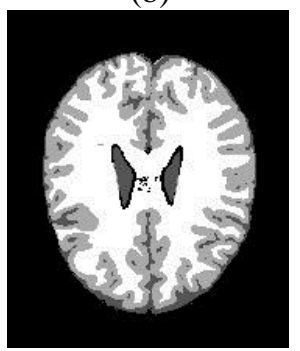

(d)

Fig. 1. BrainWeb brain MRI and ground truth: (a) BW Z93 (b) ground truth of BW Z93 (c) BW Z100 (d) ground truth of BW Z100.

Table 1. Average experimental results (of 10 runs) obtained by FCM, RFCM, and the proposed SSRFCM performed on various IBSR and BrainWeb brain MRI datasets.

Table 2. Paired $t$-tests results: based on the OSA obtained by the proposed SSRFCM versus FCM and

\begin{tabular}{|c|l|l|l|l|}
\hline $\begin{array}{c}\text { MRI } \\
\text { Data }\end{array}$ & Methods & OSA & Jaccard & Dice \\
\hline & FCM & 88.32 & 0.883 & 0.937 \\
BW Z93 & RFCM & 90.35 & 0.903 & 0.948 \\
& SSRFCM & $\mathbf{9 1 . 9 0}$ & $\mathbf{0 . 9 1 9}$ & $\mathbf{0 . 9 5 6}$ \\
\hline \multirow{3}{*}{ BW Z100 } & FCM & 87.98 & 0.879 & 0.926 \\
& RFCM & 88.89 & 0.888 & 0.931 \\
& SSRFCM & $\mathbf{9 0 . 4 7}$ & $\mathbf{0 . 9 0 4}$ & $\mathbf{0 . 9 4 7}$ \\
\hline \multirow{3}{*}{ IBSR 155 } & FCM & 92.20 & 0.922 & 0.959 \\
& RFCM & 94.49 & 0.944 & 0.974 \\
& SSRFCM & $\mathbf{9 5 . 3 9}$ & $\mathbf{0 . 9 5 3}$ & $\mathbf{0 . 9 7 6}$ \\
\hline \multirow{3}{*}{ IBSR 167 } & FCM & 92.56 & 0.925 & 0.961 \\
& RFCM & 94.94 & 0.949 & 0.974 \\
& SSRFCM & $\mathbf{9 5 . 6 1}$ & $\mathbf{0 . 9 5 6}$ & $\mathbf{0 . 9 7 7}$ \\
\hline
\end{tabular}

RFCM in terms of p-score for BrainWeb and IBSR brain MRI datasets.

\begin{tabular}{|c|c|c|}
\hline $\begin{array}{c}\text { MRI } \\
\text { Data Sets }\end{array}$ & $\begin{array}{c}\text { SSRFCM } \\
\text { Vs. } \\
\text { FCM }\end{array}$ & $\begin{array}{c}\text { SSRFCM } \\
\text { Vs. } \\
\text { RFCM }\end{array}$ \\
\hline BW Z093 & $\mathbf{1 . 4 9 3 8 e - 0 5}$ & $\mathbf{0 . 0 0 3 0}$ \\
BW Z100 & $\mathbf{3 . 0 4 1 4 e - 0 6}$ & $\mathbf{0 . 0 0 1 8}$ \\
IBSR 155 & $\mathbf{0 . 0 2 4 0}$ & $\mathbf{0 . 0 2 9 0}$ \\
IBSR 167 & $\mathbf{0 . 0 0 1 7}$ & 0.1616 \\
\hline
\end{tabular}

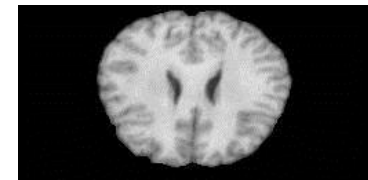

(a)

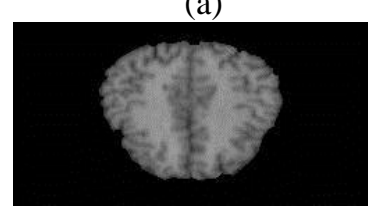

(c)

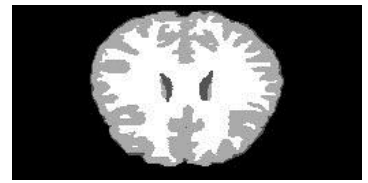

(b)

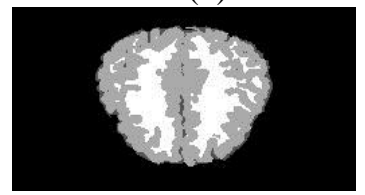

(d)
Fig. 2. IBSR brain MRI and ground truth: (a) IBSR 155 (b) ground truth of IBSR 155 (c) IBSR 167 (d) ground truth of IBSR 167.

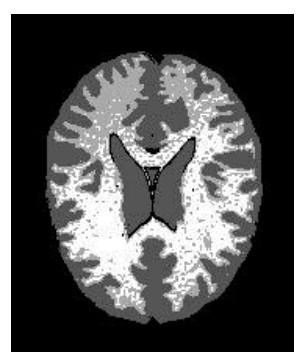

(a)

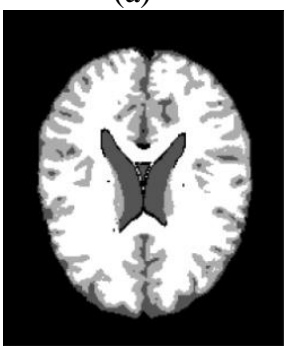

(c)

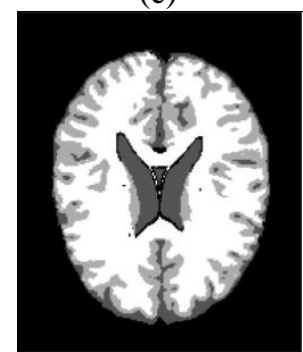

(e)

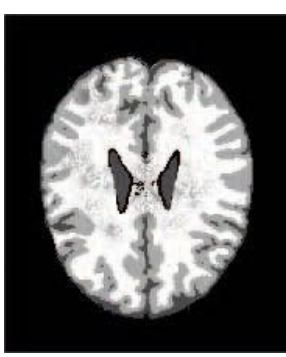

(b)

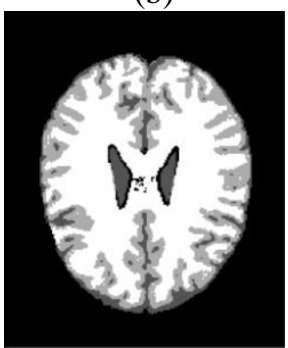

(d)

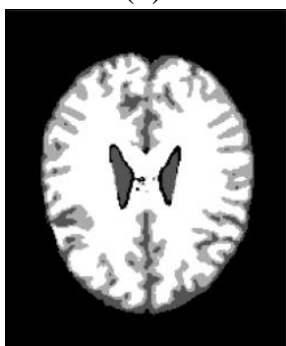

(f)
Fig. 3. Segmentation results of Fig. 1: First row ((a) BW Z93 (b) BW Z100) by FCM. Second row ((c) BW Z93 (d) BW Z100) by RFCM. Third row ((e) BW Z93 (f) BW Z100) by SSRFCM 


\section{Available online at www.ijrat.org}

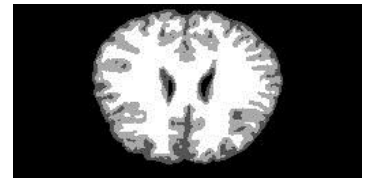

(a)

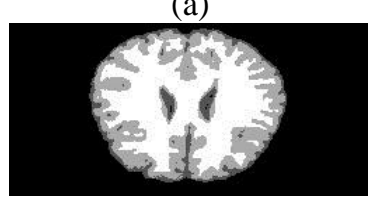

(c)

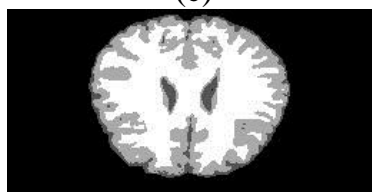

(e)

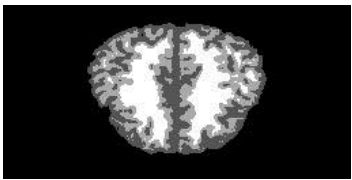

(b)

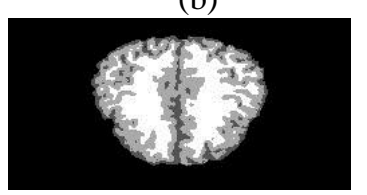

(d)

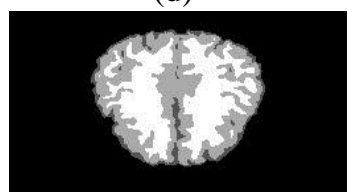

(f)
Fig. 4. Segmentation results of Fig. 1: First row ((a) IBSR 155 (b) IBSR 167) by FCM. Second row ((c) IBSR 155 (d) IBSR 167) by RFCM. Third row ((e) IBSR 155 (f) IBSR 167) by SSRFCM.

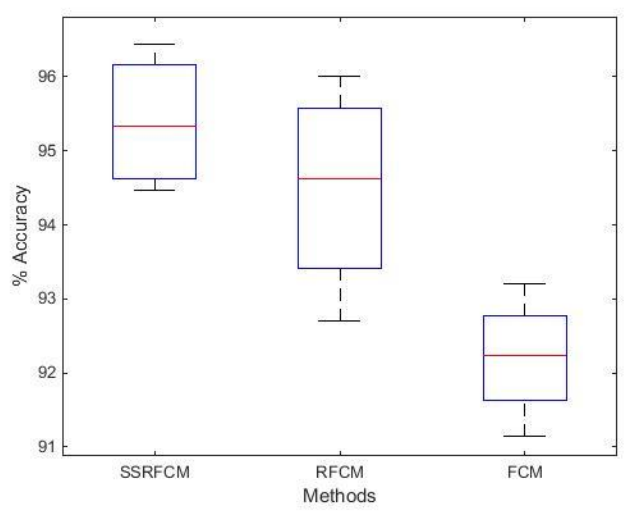

(a)

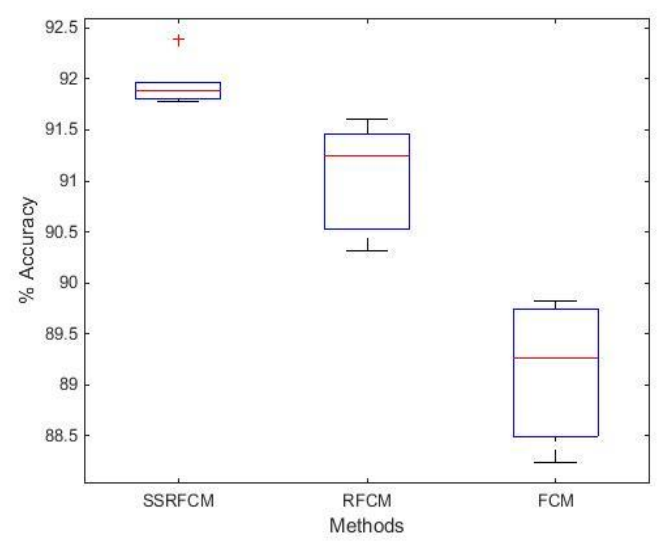

(c)

\section{CONCLUSIONS}

In modern medical diagnosis, automated systems play a major role for proper investigation of diseases and giving accurate therapy. Mostly segmentation is the first step in almost all automated diagnosing systems where MRI is concerned.

In brain (brain MRI) generally the boundaries of different regions are vague, uncertain, indiscernible and overlapping in nature. To deal with these characteristics semi-supervised rough fuzzy c-means clustering for brain MRI segmentation is proposed. Where, fuzzy set and the rough set concepts manage the above said characteristics of brain MRI. On the other hand, constraints in the form of labeled pixels are used in the method by initializing and constraining the process of clustering which guides the algorithm in the direction of a more accurate segmentation of brain regions.

Variety of benchmark brain MRI are considered from BrainWeb (freely available online at: http://brainweb.bic.mni.mcgill.ca/brainweb) and IBSR (freely available online at: https://www.nitrc.org/projects/ibsr) for the experimental purpose. Simulated results are validated using overall accuracy, jaccard, and dice coefficient.

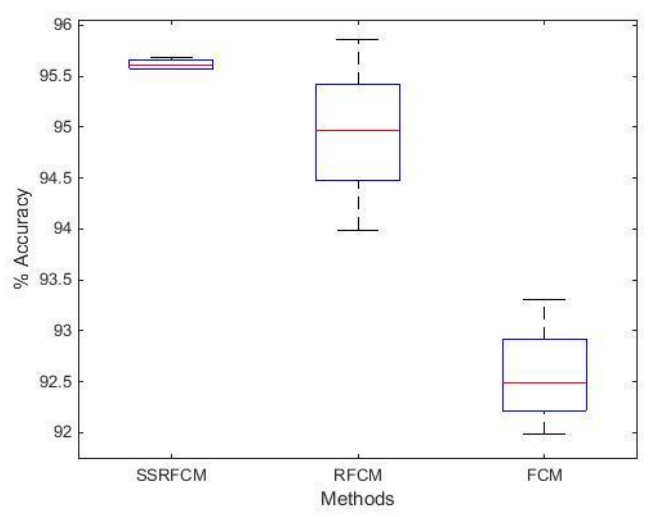

(b)

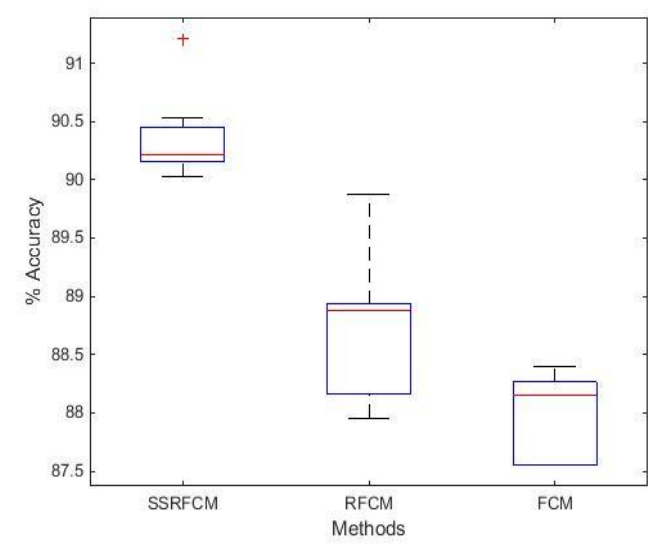

(d)

Fig. 5. Boxplots of segmentation accuracies obtained by the proposed method SSRFCM and other compared methods FCM and RFCM performed on different brain MRI datasets: (a) IBSR155, (b) IBSR167, (c) BW Z93, and (d) BW Z100. 
International Journal of Research in Advent Technology, Vol.7, No.3, March 2019

E-ISSN: 2321-9637

\section{Available online at www.ijrat.org}

Results are compared with benchmark clustering based segmentation algorithm. Statistical significance of the results produced by the proposed SSRFCM method is tested using paired $t$-test. Box-plots are also shown to confirm the better results by the proposed method compared to the counterpart methods. From the segmentation accuracy, $t$-test results, box-plots as well as from the visualization quality of the segmented results it can be argued that the proposed method with constraints significantly enhanced the segmentation accuracy of brain MRI segmentation with a reasonably small amount of supervision.

\section{REFERENCES}

[1] Haidekker, M. A. (2013): Medical Imaging Technology. Springer, New York, first edition.

[2] Balafar, M. A.; Ramli, A. R.; Saripan, M. I.; and Mashohor, S. (2010): Review of brain MRI image segmentation methods. Artificial Intelligence Review, 33(4):261-274.

[3] Suetens, P. (2002): Fundamentals of Medical Imaging. Cambridge University Press, Cambridge, UK, first edition.

[4] Maji, P.; and Pal, S. K. (2012): Rough-fuzzy pattern recognition: applications in bioinformatics and medical imaging. John Wiley \& Sons, Inc, New Jersey and Canada.

[5] Bauer, S.; Wiest R.; Nolte, L. P.; and Reyes, M. A. (2013): survey of MRI based medical image analysis for brain tumor studies. Physics in Medicine and Biology, 58(13):97-129.

[6] Bishop, C. M. (2006): Pattern Recognition and Machine Learning (Information Science and Statistics). Springer, Verlag New York, first edition.

[7] Gonzalez, R. C.; and Woods, R. E. (2017): Digital Image Processing. Pearson, India, third edition.

[8] Theodoridis, S.; and Koutroumbas, K. (2009): Pattern Recognition. Academic Press, New York, fourth edition.

[9] Manousakes, I. N.; Undrill, P. E.; and Cameron, G. G. (1998): Split and merge segmentation of magnetic resonance medical images: Performance evaluation and extension to three dimensions. Computers and Biomedical Research, 31(6):393412.

[10] Subudhi, B. N.; Thangaraj, V.; Sankaralingam, E.; and Ghosh, A. (2016): Tumor or abnormality identification from magnetic resonance images using statistical region fusion based segmentation. Magnetic Resonance Imaging, 34(9):1292-1304.

[11] Banerjee, S.; Mitra, S.; and Umashankar, B. (2016): Single seed delineation of brain tumor using multi-thresholding. Information Sciences, 330(4):88-103.

[12] Rajapakse, J. C.; Giedd, J. N.; and Rapoport, J. L. (1997): Statistical approach to segmentation of single channel cerebral MR images. IEEE Transactions on Medical Imaging, 16(2):176-186.

[13] Singleton, H. R.; and Pohost, G. M. (1997): Automatic cardiac MR image segmentation using edge detection by tissue classification in pixel neighborhoods. Magnetic Resonance in Medicine, 37(3):418-424.

[14] Banerjee, A.; and Maji, P. (2016): Roughprobabilistic clustering and hidden markov random field model for segmentation of HEp-2 cell and brain MR images. Applied Soft Computing, 46(C):558-576.

[15] Saha, S.; and Bandyopadhyay, S. (2007): MRI brain image segmentation by fuzzy symmetry based genetic clustering technique. In Proceedings of the IEEE Congress on Evolutionary Computation (CEC), pages 44174424.

[16]Maji, P.; and Roy, S. (2015): Rough-fuzzy clustering and unsupervised feature selection for wavelet based MR image segmentation. PLOS ONE, 10(4):1-30.

[17] Vishnuvarthanana, G.; Rajasekaran, M. P.; Subbaraj P.; and Vishnuvarthanan, A. (2016): An un-supervised learning method with a clustering approach for tumor identification and tissue segmentation in magnetic resonance brain images. Applied Soft Computing, 38(C):190-212.

[18] Sarkara, J. P.; Saha I.; and Maulik U. (2016): Rough possibilistic type-2 fuzzy c-means clustering for MR brain image segmentation. Applied Soft Computing, 46(C):527-536.

[19] Ravishankar, S.; and Bresler, Y. (2011): MR image reconstruction from highly under sampled k-space data by dictionary learning. IEEE Transactions on Medical Imaging, 30(5):10281041.

[20] Dahshan, E. S. A. E.; Mohsen, H. M.; Revett K.; and Salem, A. B. M. (2014): Computer-aided diagnosis of human brain tumor through MRI: A survey and a new algorithm. Expert Systems with Applications, 41(11):5526-5545.

[21]Liu, J.; Li, M.; Wang, J.; Wu, F.; Liu, T.; and Pan, Y. (2014): A survey of MRI based brain tumor segmentation methods. Tsinghua Science and Technology, 19(6):578-595.

[22] Gordillo, N.; Montseny, E.; and Sobrevilla, P. (2013): State of the art survey on MRI brain tumor segmentation. Magnetic Resonance Imaging, 31(8):1426-1438.

[23] Basu, S.; Banerjee, A.; and Mooney R. J. (2004): Active Semi-Supervision for Pairwise Constrained Clustering. Proceedings of the SIAM International Conference on Data Mining, pp. 333-344.

[24] Basu, S.; Banerjee, A.; and Mooney R. J. (2002): Semi-supervised clustering by seeding. Proceedings of the $19^{\text {th }}$ international conference on machine learning, pp. 19-26.

[25]Zadeh, L. A. (1965): Fuzzy sets. Information and Control, 8(3):338-353.

[26] Pawlak, Z. (1982): Rough sets. International Journal of Computer and Information Science, 11(5):341-356.

[27]Pawlak, Z. (1991): Rough Sets: Theoretical Aspects of Reasoning About Data. Kluwer, Dordrecht, Netherlands. 
[28] Bezdek, J. C.; Ehrlich, R.; and Full W. (1984): FCM: the fuzzy c-means clustering algorithm. Computer and Geosciences, 10(2-3):191-203.

[29] Cai, W.; Chen, S.; and Zhang, D. (2006): Fast and robust fuzzy c-means clustering algorithms incorporating local information for image segmentation. Pattern Recognition, 40 (2007): $825-838$.

[30] Collins, D. L.; Zijdenbos, A. P.; Kollokian, V.; Sled, J. G.; Kabani, N. J.; Holmes, C. J.; and Evans, A. C. (1998): Design and construction of a realistic digital brain phantom. IEEE Transactions on Medical Imaging, 17(3):463-468.

[31] Rohlfing, T. (2012.): Image similarity and tissue overlaps as surrogates for image registration accuracy: Widely used but unreliable. IEEE Transactions on Medical Imaging, 31(2):153-163.

[32] Maji, P.; and Pal, S. K. (2007): Rough set based generalized fuzzy c-means algorithm and quantitative indices. IEEE Trans. Systems, Man, and CyberneticsPart B: Cybernetics, 37(6):15291540.

[33] Maji, P.; and Pal, S. K. (2007): RFCM:a hybrid clustering algorithm using rough and fuzzy sets. Fundamenta Informaticae, 80(4):475-496.

[34] Han, J.; Kamber, M.; and Pei, J. (2011): Data Mining: Concepts and Techniques. Third edition.

[35] Tukey, J. W. (1977): Exploratory Data Analysis. Addison-Wesley.

[36]Rice, J. A. (2006): Mathematical Statistics and Data Analysis. Advanced series. Cengage Learning. 